\title{
Cerebral White Matter Hyperintensity in T2 Weighted Magnetic Resonance Images of Elderly Patients: Correlation with Mental Status
}

\author{
- Niraj Regmi' • Abu Saleh Mohiuddin² $\bullet$ Abu Taher ${ }^{2} \bullet$ Mahfuz Ara Ferdousi' $^{2}$
}

Submitted 9 December 2020

$\equiv \quad$ Niraj Regmi

regmi.niraj@gmail.com

iD https://orcid.org/0000-0003-0484-9637

${ }^{1}$ Department of Radiology and Imaging, B. P. Koirala Institute of Health Sciences, Dharan, Nepal

2 Department of Radiology and Imaging, BIRDEM General Hospital, Dhaka, Bangladesh

\section{Citation}

“ Regmi N, Mohiuddin AS, Taher A, Ferdousi M. Cerebral white matter hyperintensities in $T 2$ weighted magnetic resonance images of elderly patients- correlation with their mental status. JBPKIHS. 2021;4(1):26-31.

doi https://doi.org/10.3126/jbpkihs.v4i1.36107

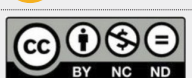

This work is licensed under a Creative Commons Attribution NonCommercial 4.0 International License.
Accepted 27 March 2021

Published 30 June 2021
Background: White matter hyperintensity (WMH), focal and/ or diffuse areas of hyperintense signals on T2 weighted magnetic resonance imaging (MRI), is the most common incidental finding in elderly patients. However, its clinical significance is usually overlooked.We aimed to find out the correlation between cerebral WMH in MRI with the mental status of elderly patients, assessed by Mini-Mental Status Examination (MMSE) score.

Methods: This cross-sectional study was conducted for two years on eighty eligible elderly patients (> 60 years) referred to the Department of Radiology and Imaging for MRI of the brain. Demographic variables like age and sex, MMSE score, and MRI variables like location and the number of WMHs were studied. The Pearson's correlation coefficient was used to assess the correlation between the extent of periventricular WMH and MMSE score.

Results: A significant negative correlation $(r=-0.78 ; p<0.00 I)$ was found between MMSE and the extent of periventricularWMH. A significant negative correlation was also found when periventricular hyperintensities were evaluated individually for frontal caps $(r=-0.68$; $<0.001)$, band opacities $(r=$ $-0.55 ; p<0.00 I)$ and occipital caps $(r=-0.59 ; p<0.00 I)$. However, subcortical WMH was not significantly corelated with MMSE score $(r=+0.02, p=0.09)$. Conclusion: The extent of periventricular WMH seen in the brain MRI is significantly correlated with a cognitive decline in elderly subjects. However, no such correlation exists between subcortical WMH and mental status.

Keywords: Fluid attenuation inversion recovery (FLAIR); Mini-mental status examination (MMSE) score; T2 weighted magnetic resonance imaging (T2W MRI);White matter hyperintensities (WMH)

\section{Declarations}

Ethics approval and consent to participate: Ethical approval was obtained from Institute Review Committee of BIRDEM (Ref. No. - BADAS-ERC/ $\mathrm{EC} / 17 / 0 / 4)$ and written informed consent was taken from all the patients.

Consent for publication: Not applicable

Availability of data and materials: The datasets used and/or analyzed during the current study are available from the corresponding author on reasonable request. All relevant data are within the manuscript and its supporting information files.

Competing interest: None
Funding: None

Authors' contributions: NR: concept, data collection, and manuscript preparation.ASM: concept, data collection, and manuscript editing/ review. MAT: design, statistical analysis, and manuscript review/ approval. MAF: critical review of manuscript and approval. All the authors have read and approved the final manuscript. Acknowledgement: None 
$\mathrm{W}$ hite matter hyperintensity (WMH), often found on Magnetic Resonance Imaging (MRI) scans of elderly people, is attributed to degenerative changes of long penetrating arteries. WMH burden (i.e., volume or severity) is negatively associated with performance across a range of cognitive tests, particularly those involving executive functioning, processing speed, and attention [1]. The prevalence has been reported to range from 5 to $90 \%$, depending on the study design, population, and rating scales [2].

WMH is considered the tip of an iceberg, reflecting an underlying process with no visible boundaries at MRI. WMH has been correlated with cognitive, behavioral, gait, and urinary impairment and have been shown to progress over time [3]. Periventricular, but not subcortical, WMH has been related to atherosclerosis and cognitive impairment, hence illustrating the importance of differentiating between WMH at different locations [4]. A recent study reports an association of cerebral WMH burden on MRI with a DNA methylation-based biomarker of aging, termed DNA methylation age acceleration, which represents the deviation of the DNA methylation-predicted age from the chronologic age [5].

In MRI, T2 weighted (T2W)/ Fluid attenuation Inversion Recovery (FLAIR) overestimates periventricular and perivascular WMH compared to histopathologically confirmed demyelination. The relatively high concentration of interstitial water in the periventricular/ perivascular regions due to the increased blood-brain-barrier permeability and plasma leakage in brain aging may evoke T2W/ FLAIR WMH despite relatively mild demyelination [6].

The Mini-Mental State Examination (MMSE) score of less than 24 is commonly used to reflect cognitive impairment $[7,8]$. Despite being a rough measure of global cognitive function, the severity of WMH is associated with the rate of decline of the MMSE score and with the MMSE score at the time of imaging [4].

WMH is a poor prognostic marker in stroke outcomes. Recent studies have shown that WMH coexists with ischemic stroke in patients with large artery disease like intracranial atherosclerosis, however, the relationship between WMH and large vessel disease is still unclear [9].

WMH also contributes to Alzheimer's disease dementia; preventive strategies to reduce cardiovascular disease and WMH could decrease or delay the incidence of the onset of dementia [10]. We aimed to find out the correlation between the degree of cerebral WMH evaluated by MRI with the mental status of elderly subjects assessed by MMSE score.

\section{METHODS}

This cross-sectional study was done on eighty patients in the Department of Radiology and Imaging, BIRDEM General Hospital, Dhaka, Bangladesh from July 2013 to June 2015. A purposive sampling technique was applied. Elderly patients ( $>60$ years) who were referred for a medical checkup and advised for an MRI of the brain without manifestations of neurological deficit were enrolled. Uncooperative or unconscious patients and those with suspected stroke or intracranial neoplasm were excluded. The study was approved by the Institutional Review Committee of BIRDEM and written informed consent was taken from all the patients.

After explaining to the patients all the necessary information regarding the research study, data was collected in a structured sheet. First, a researcher examined the MMSE score of the patient. Then the MRI of the brain was examined by the same researcher which was then cross-examined by a senior radiologist with 12 years of experience in MRI. To avoid bias, a hard copy of MRI of the brain of the same patient was examined by another senior radiologist with 10 years of experience who was unaware of the MMSE score. The MRI findings were recorded for correlation with the MMSE score previously obtained. Variables assessed included demographics such as age and sex of the patient, the MMSE score along with the MRI variables such as location and number of lesions, and size and location of the largest lesion.

\section{MINI-MENTAL STATUS EXAMINATION}

The MMSE is a useful screening questionnaire to detect cognitive impairment. A score of less than 24 out of 30 indicates cognitive impairment [8]. It is an eleven-question measure that tests five areas of cognitive function: orientation, registration, attention, recall, and language (Table 1). Patients with an MMSE score of 24 and above were classified as normal while those below 24 had a cognitive decline.

\section{SCANNING TECHNIQUE}

The MRI of the brain was performed on a Neusoft $0.35 \mathrm{~T}$ scanner (Neusoft Medical Systems Co., 
Limited, China). The patient was placed supine on the examination table and a proper head coil was used to send and receive radiofrequency pulses. The scanning protocol included a series of axial T2 weighted images (TR- $4600 \mathrm{~ms}$; TE- $132 \mathrm{~ms}$; Flip angle- 90 ${ }^{\circ}$, FLAIR images (TR- $4234.6 \mathrm{~ms}$; TE- 88.0 ms; Flip angle- 90 ${ }^{\circ}$; Inversion TI- $1600 \mathrm{~ms}$ ), and T1 weighted images (TR22.0 ms; TE- $7.5 \mathrm{~ms}$; Flip angle- $30^{\circ}$ ). Sections were 6.0 $\mathrm{mm}$ thick with an interslice gap of $7.2 \mathrm{~mm}$. Hardcopies were printed by a thermal printer. Periventricular and subcortical WMHs were considered present if visible as hyperintense on both T2 weighted and FLAIR images, without prominent hypointensity on $\mathrm{T} 1$ weighted images.

\section{INTERPRETATION OF MRI OF BRAIN}

WMH Rating Scale: The presence, severity, and lo- cation of morphological brain characteristics were rated according to the protocol designed for the Rotterdam Scan Study [11]. WMH was considered present in case of hyperintense lesions on both FLAIR and T2-weighted images but not hypointense on T1-weighted images or not showing bright signal in diffusion-weighted sequences. When the largest diameter of the WMH was adjacent to the ventricle, it was considered as periventricular, otherwise as subcortical.

Periventricular WMH was rated semi-quantitatively by visual rating scale as o (none), 1 (pencil-thin lining), 2 (smooth halo), or 3 (large confluent). Three separate regions that were considered were adjacent to frontal horns (frontal caps), adjacent to the wall of the lateral ventricles (bands), and adjacent to the occipital horns (occipital caps). The overall degree of periventricular WMH was calculated by adding up the scores
Figure 1: Periventricular WMH (a. $\mathrm{T} 2 \mathrm{~W}$ and $\mathrm{b}$. FLAIR images). (a)

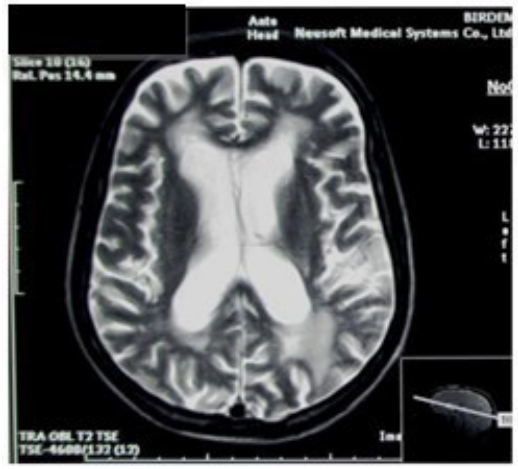

(b)

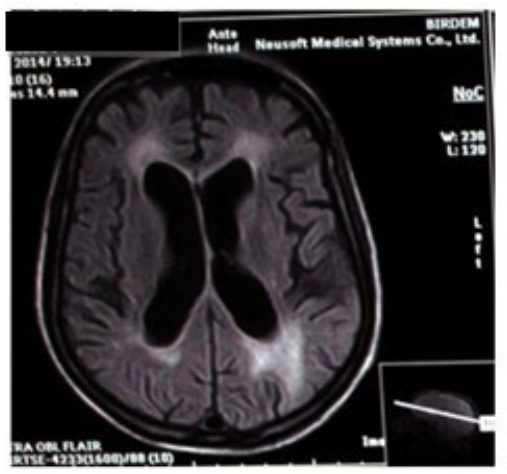

Figure 2: Periventricular band and subcortical WMH (a. FLAIR, and b. T2W images) (a)

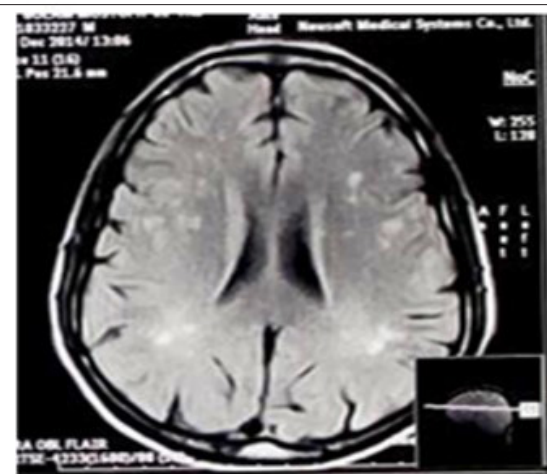

(b)

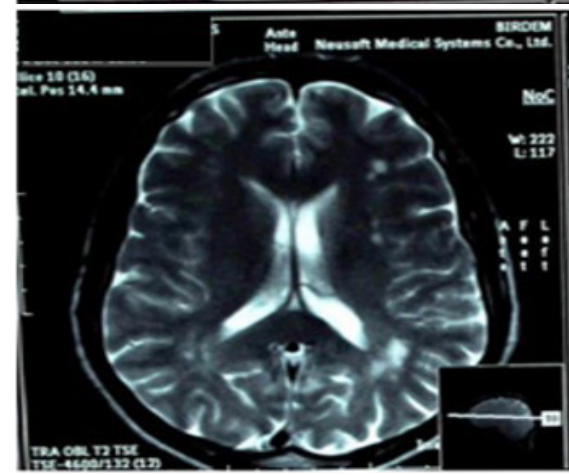

itauntion

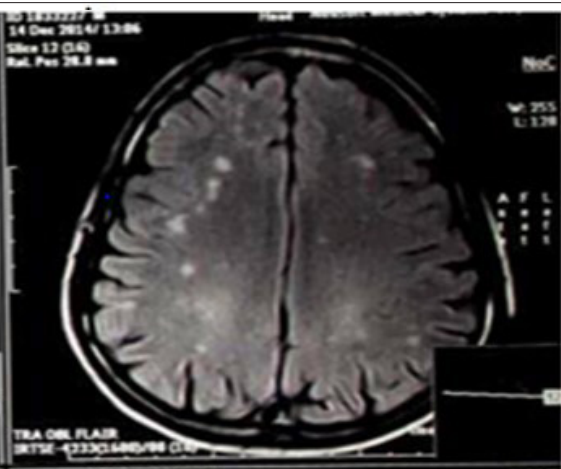

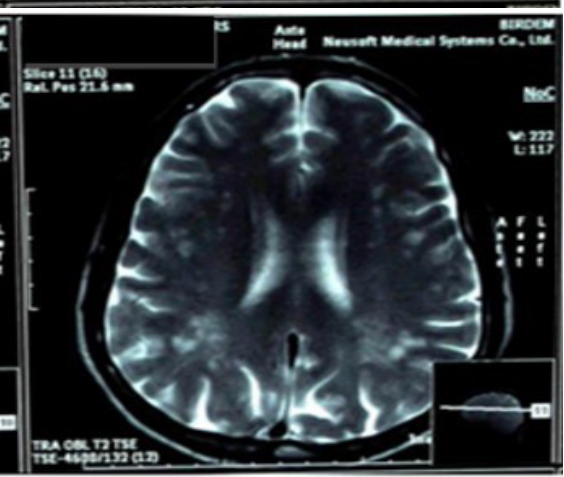


for the three separate categories (range: o - 9) (Fig. 1 and 2).

All the collected data were compiled on a master chart first. Then the data were organized by using a scientific calculator and standard statistical formulas. The percentage was calculated to find out the proportion of the findings. Further statistical analyses of the data were done by the software Statistical Packages for Social Science version 11.5. The Pearson's correlation coefficient was used to evaluate the relationship among different WMH and MMSE score. A value of $\mathrm{p}<0.05$ was considered statistically significant. Variables were demonstrated as mean \pm SD for ages and mean \pm SEM for all the remaining parameters.

\section{RESULTS}

$\mathrm{A}$

total of 80 consecutive eligible subjects were enrolled, among whom 49 (61.3\%) were males. The mean age was $68.6 \pm 7.2$ years (range: 61 to 90 years). There was no missing data.

The MMSE score ranged from 15 to 30. The majority $(55 \%)$ of patients had MMSE score $<24$. The overall degree of periventricular WMHs score ranged from 3 to 9 and the score 4 had the highest frequency (25\%). A significant negative correlation ( $\mathrm{r}=-0.78$; $\mathrm{p}<$ o.001) was found between MMSE score and the extent of periventricular WMH at MRI of the brain (Fig. 3).

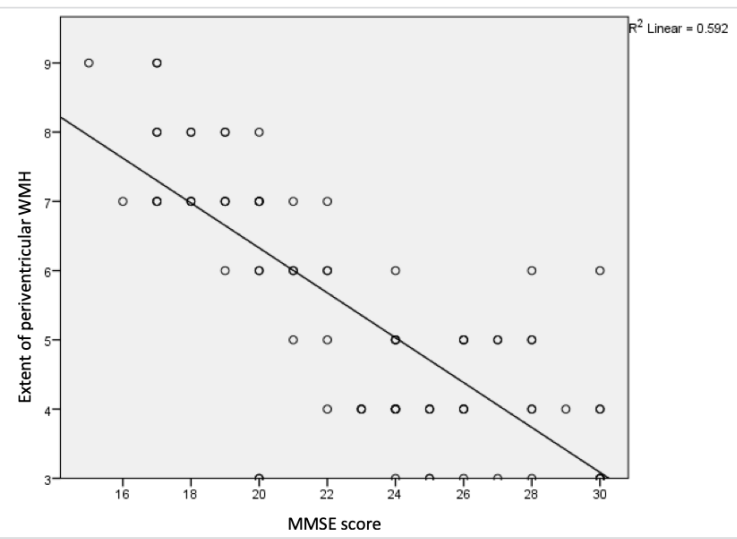

Figure 3: Correlation between MMSE score and extent of periventricular WMH

The MMSE score was independently correlated with hyperintensities in the frontal periventricular white matter $(\mathrm{r}=-0.68 ; \mathrm{p}<0.001)$, periventricular white matter band opacities $(\mathrm{r}=-0.55$; $\mathrm{p}<0.001)$ and periventricular occipital white matter opacities $(\mathrm{r}=$ -0.59; p < 0.001) (Fig. 4).
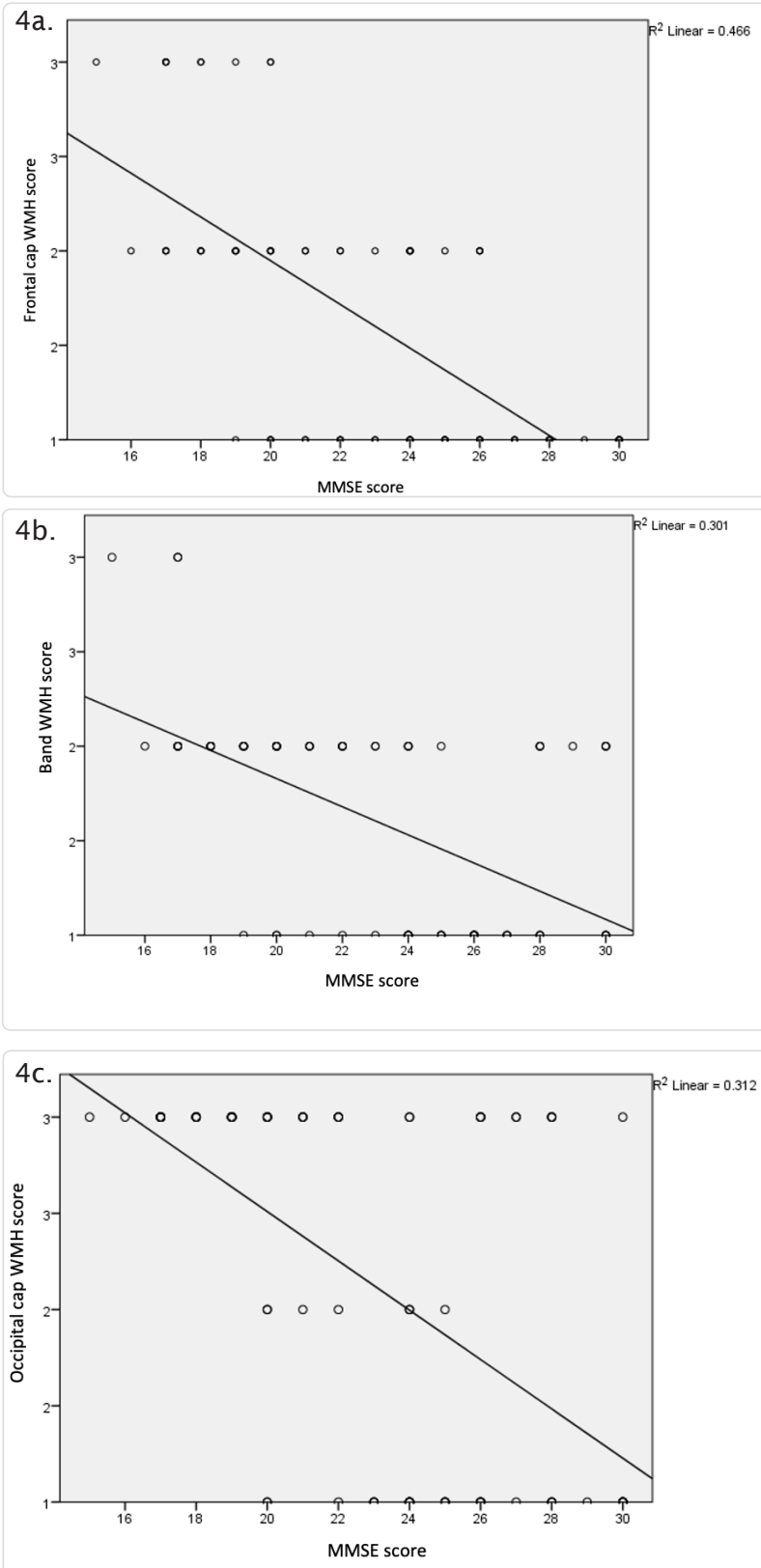

Figure 4: Correlation between MMSE score and a. frontal cap WMH, b. band WMH, and c. occipital WMH

No correlation ( $\mathrm{r}=0.02 ; \mathrm{p}=0.09$ ) was found between the MMSE score and the largest size of subcortical lesion at MRI of the brain (Fig. 5).

\section{DISCUSSION}

T ${ }^{\mathrm{MH}}$ reflects accumulating white matter damage with aging and impair cognition. More WMHs have been shown elsewhere to be significantly associated 


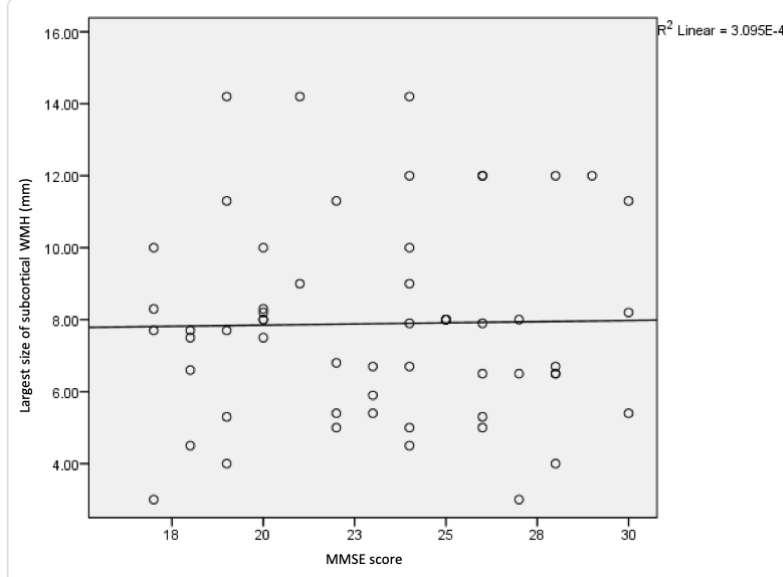

Figure 5: Correlation between MMSE score and the largest size of the subcortical WMH

with lower cognitive ability [12]. In this study, the incidence of periventricular WMH was more than that of subcortical WMH and all of the patients had periventricular WMH while subcortical WMH was present only in combination with periventricular WMH in $71.3 \%$ of patients. In another study, the percentage of periventricular WMH (74\%) was also more than subcortical lesions (68\%) [13].

Periventricular WMH was rated semi-quantitatively and three separate regions were considered which were frontal caps, bands, and occipital caps. The overall degree of periventricular WMH was calculated by adding up scores for three separate categories (range: 0-9). A study done by de Leeuw et al. was based on similar methods for assessing WMH [2].

When the scores of MMSE of our patients were correlated with the extent of periventricular WMH at MRI of the brain, and then separately for frontal caps, band opacities, and occipital cap, a significant negative correlation was found between these parameters. However, the negative correlation between peri-ventricular lesion and MMSE is less likely to be affected by lobar predilection (occipital/ frontal or band hyper-intensity). Among the three, frontal periventricular WMH showed a relatively more negative correlation than the other two. However, studies showing the correlation at an individual level are lacking and more research needs to be done.

A general pattern of age-related preservation and decline emerges indicating that the prefrontal WMH is most susceptible to the influence of age. Studies that combine MRI with cognitive measures suggest that such age-related reductions in white matter integrity may produce a disconnection state that underlies some of the age-related performance declines in age-sensitive cognitive domains [14].

The current study found that mental status was not affected by subcortical WMH. Periventricular, but not subcortical WMH, has been related to atherosclerosis, and cognitive impairment has been related to periventricular $\mathrm{WMH}$, but not to subcortical $\mathrm{WMH}$, illustrating the importance of differentiating between WMH at different locations [4].

Similarly, another study has reported that only periventricular WMH and not subcortical WMH was independently associated with cognitive decline [15]. It was more notable for executive functions, mental and motor speed [15]. The educational background is greatly dependent on the environment during childhood, and therefore some researchers also consider that the childhood environment may influence future cognitive impairment [8].

Investigators have found a gradation of flow and blood volume in WMH that decrease from normal tissue on the periphery to reduced values in the center of a lesion [16]. This may be the reason behind the decline of cognition associated with WMH seen in our patients.

Our study aimed at dealing with common incidental findings of WMH in MRI of the brain. Correlation with cognitive state emphasizes that this finding should not be neglected. Our study has some limitations. First, the sample size was small as most of the elderly patients referred for MRI of the brain had coexisting findings; hence, they could not be enrolled. Second, this study was carried out in a single center. Third, the MR scanner was less powerful (0.35T).

\section{CONCLUSION}

Cognitive decline in elderly subjects is significantly correlated with the extent of periventricular white matter hyperintensity at MRI of the brain. However, the mental status of the elderly people is poorly correlated with subcortical white matter hyperintensity. This important finding may assist clinicians with proper counseling and additional management. 


\section{References}

I. Brickman AM, Siedlecki KL, Muraskin J, Manly J], Luchsinger JA, Yeung L-K, et al. White matter hyperintensities and cognition: testing the reserve hypothesis. Neurobiol Aging. 20 I I;32(9): 158898. DOI: 10.1016/j.neurobiolaging.2009.10.013

2. De Leeuw FE, de Groot JC, Achten E, Oudkerk M, Ramos LM, Heijboer R, et al. Prevalence of cerebral white matter lesions in elderly people: a population based magnetic resonance imaging study. The Rotterdam Scan Study. J Neurol Neurosurg Psychiatry. 2001;70(I):9-I4. DOI: I0.1 I36/jnnp.70.1.9

3. Sarbu N, Shih RY, Jones RV, Horkayne-Szakaly I, Oleaga L, Smirniotopoulos JG. White matter diseases with radiologic-pathologic correlation. Radiographics. 2016;36(5):1426-47. DOI: I0.II48/ rg.2016160031

4. De Groot JC, De Leeuw FE, Oudkerk M, Van Gijn J, Hofman A, Jolles J, et al. Periventricular cerebral white matter lesions predict rate of cognitive decline. Ann Neurol. 2002;52(3):335-4I. DOI: 10.1002/ana. 10294

5. Raina A, Zhao X, Grove ML, Bressler J, Gottesman RF, Guan W, et al. Cerebral white matter hyperintensities on MRI and acceleration of epigenetic aging: the atherosclerosis risk in communities study. Clin Epigenetics. 2017;9(I):2I. DOI: I0.1 I86/s/3148-0160302-6

6. Haller S, Kovari E, Herrmann FR, Cuvinciuc V, Tomm A-M, Zulian $\mathrm{GB}$, et al. Do brain T2/ FLAIR white matter hyperintensities correspond to myelin loss in normal aging? A radiologic-neuropathologic correlation study. Acta Neuropathol Commun. 20I3; I (I): I4 DOI: I0.I I86/205 I-5960-I-I4

7. Folstein MF, Folstein SE, McHugh PR. “Mini-mental state”. A practical method for grading the cognitive state of patients for the clinician. J Psychiatr Res. 1975; 12(3): 189-98. DOI: 10.1016/00223956(75)90026-6

8. Koga H, Yuzuriha T, Yao H, Endo K, Hiejima S, Takashima Y, et al. Quantitative MRI findings and cognitive impairment among community dwelling elderly subjects. J Neurol Neurosurg Psychiatry. 2002;72(6):737-4I.DOI: I0.I I 36/jnnp.72.6.737

9. Habes M, Erus G, Toledo JB, Zhang T, Bryan N, Launer LJ, et al. White matter hyperintensities and imaging patterns of brain age- ing in the general population. Brain. 20I6; I39(Pt 4): I |64-79. DOI: 10.1093/brain/aww008

10. Nam KW, Kwon HM, Jeong HY, Park JH, Kim SH, Jeong SM, et al. Cerebral white matter hyperintensity is associated with intracranial atherosclerosis in a healthy population. Atherosclerosis. 2017;265:179-83. DOI: 10.1016/j.atherosclerosis.2017.09.010

II. Ikram MA, van der Lugt A, Niessen WJ, Krestin GP, Koudstaal PJ, Hofman A, et al. The Rotterdam Scan Study: design and update up to 20I2. Eur J Epidemiol. 20I I;26(I0):8I I-24. DOI: 10.1007/ sl0654-0I I-9624-z

12. Valdés Hernández Mdel C, Booth T, Murray C, Gow AJ, Penke L, Morris Z, et al. Brain white matter damage in aging and cognitive ability in youth and older age. Neurobiol Aging. 201 3;34(I 2):27407. DOI: I0.1016/j.neurobiolaging.2013.05.032

13. Silbert LC, Nelson C, Howieson DB, Moore MM, Kaye JA. Impact of white matter hyperintensity volume progression on rate of cognitive and motor decline. Neurology. 2008;7 I (2): I08- I3. DOI: 10.12/2/0I.wnl.00003/6799.869|7.37

14. Gunning-Dixon FM, Brickman AM, Cheng JC,Alexopoulos GS.Aging of cerebral white matter: a review of MRI findings. Int J Geriatr Psychiatry. 2009;24(2): 109-17. DOI: 10.1002/gps.2087

I5. Van Harten B, Oosterman J, Muslimovic D, van Loon BJ, Scheltens P,Weinstein HC. Cognitive impairment and MRI correlates in the elderly patients with type 2 diabetes mellitus. Age Ageing. 2007;36(2): I64-70. DOI: I0.1093/ageing/afl I 80

16. Kim KW, MacFall JR, Payne ME. Classification of white matter lesions on magnetic resonance imaging in elderly persons. Biol Psychiatry. 2008;64(4):273-80. DOI: 10.1016/j.biopsych.2008.03.024 